\title{
A Model of Bridge Choice Across the Mississippi River in Minneapolis
}

\author{
Carlos Carrion* $^{*} \quad$ David Levinson ${ }^{\dagger}$
}

May 15, 2011

\begin{abstract}
On September 18th 2008, a replacement for the previously collapsed I-35W bridge opened to the public. Consequently, travelers were once again confronted with the opportunity to find better alternatives. The traffic pattern of the Minneapolis road network was likely to readjust, because of the new link addition. However, questions arise about the possible reasons (or components in the route choice process) that are likely to influence travelers crossing the Mississippi, who had to choose among several bridge options, including the new I-35W bridge. A statistical model of bridge choice is specified and estimated employing weighted-least squares logit, and using Global Positioning System (GPS) data and web-based surveys collected both before and after the replacement bridge opened. In this way the proportion of I-35W trips can be estimated depending on the assigned values of the explanatory variables, which include: statistical measures of the travel time distribution experienced by the subjects, alternative diversity, and others. The results showed that travel time savings and reliability were the main reasons for choosing the new I-35W bridge.
\end{abstract}

Keywords: GPS, route choice, I-35W bridge, wls logit.

*Graduate Student, University of Minnesota, Department of Civil Engineering, carri149@umn.edu

${ }^{\dagger}$ RP Braun-CTS Chair of Transportation Engineering; Director of Network, Economics, and Urban Systems Research Group; University of Minnesota, Department of Civil Engineering, 500 Pillsbury Drive SE, Minneapolis, MN 55455 USA, dlevinson@umn.edu, http:// nexus.umn.edu 


\section{Introduction}

In principle, travelers (if necessary) adapt to network changes (e.g. link closures, link additions) depending on their current acquired spatial information.Travelers' responses may vary according to the temporal duration and spatial occupancy of network changes. For example, closure of a residential neighborhood street may not require travel behavior adjustments for most travelers, in contrast to the closure of a major highway section. Furthermore, potential travelers' responses include:

1. switching routes;

2. canceling trips;

3. rescheduling activities;

4. using other travel modes;

5. finding alternative location of activities; and others.

Research on travelers' behavioral responses to network changes due to large-scale disruption has been limited, and consequently not many studies exist because of their unusual nature (Giuliano and Golob, 1998). Typically, network disruptions can be divided in two categories: planned and unplanned. The former are generally due to road construction or maintenance work (e.g. 1999 closure of the Centre Street Bridge in Calgary (Hunt and Stefan, 2002)), transit strikes (e.g. 1981 and 1986 strikes in Orange County (Ferguson, 1992); 2003 strike in Los Angeles (Lo and Hall, 2006); see van Exel and Rietveld (2001) for a review), major events (e.g. 2000 Olympic Games in Sydney (Hensher and Brewer, 2002); 2004 Olympic Games in Athens (Dimitriou et al., 2006)), and others. In contrast, the latter are usually attributed to natural disasters (e.g. 1989 Loma Prieta Earthquake (Tsuchida and Wilshusen, 1991); 1994 Northridge Earthquake (Wesemann et al. (1996) and Giuliano and Golob (1998)); 1995 Kobe Earthquake (Chang and Nojima, 2001)), structural failures (e.g. 2007 I-35W Bridge collapse in Minneapolis (Zhu et al., 2010)), and other severe non-recurrent events. Furthermore, this study focuses on the events after a network's major link is removed suddenly, and later on restored. These events are the collapse of the I-35W Bridge on August 1st 2007 in Minneapolis, and the reopening of the new I-35W Bridge on September 18th 2008. In the first case, travelers were forced to respond by exploring the network, and by adjusting their travel behavior according to their experience and other external information sources. In the second case, travelers were given another opportunity to explore new routes, and to decide if there are any benefits in switching to other alternatives. Consequently, the period of interest for this study is after the new I-35W bridge is open to the public, and the alternatives of interest are the bridges located along the Mississippi river near the city of Minneapolis.

It is clear that the study of travel behavior during unforeseen disruptions is the main theme in this article. Therefore, a bridge choice model is built based on data collection efforts conducted during the period between August and December of 2008. These efforts included the collection of Global Positioning System (GPS) tracking data, and web-based surveys. In addition, the travel behavior process is studied from a bridge selection reference frame; this allows for studying solely the swapping behavior of travelers (i.e. choosing I-35W Bridge vs. Other alternatives) and the possible significant explanatory factors behind them (e.g. travel time). A review of the effects 
1 of the I-35W collapse can be found in Zhu et al. (2010). This study is organized as follows: A data section presents the data collection techniques, the analysis methodology employed, and 3 descriptive statistics of the sample; the bridge choice model and its results are discussed in the subsequent section; and the last section concludes the article.

\section{Data}

\subsection{Recruitment}

7 Subjects were recruited through announcements posted in different media including: Craigslist.org,

1. Age between 25-65,

2. Legal driver,

3. Full-time job and follow a "regular" work schedule

4. Travel by driving alone

5. Likelihood of being affected by the reopening of the new I-35W Mississippi River bridge.

The possible list of potential subjects was provided to Dr. Randall Guensler at the Georgia Institute of Technology and the subcontractor Vehicle Monitoring Technologies (VMTINC), who managed this field data collection effort. Also, a local subcontractor (MachONE) was employed to instrument the subjects' vehicles with GPS devices two weeks before the new I-35W bridge reopened. These GPS devices recorded the coordinates of the instrumented vehicle at every second between engine-on and engine-off events. The coordinates log collected by the GPS was transmitted to the server in real time through wireless communication. The subjects remained instrumented for 13 weeks without following any instructions with the exception of filling periodic surveys.

In parallel, the authors and others affiliated with the University of Minnesota conducted another GPS-based data collection effort. Other potential subjects (randomly selected from the original pool) were instrumented with logging-type GPS devices (QSTARZ BT-Q1000p GPS Travel Recorder powered by DC output from in-vehicle cigarette lighter) also approximately two weeks before the replacement I-35W bridge opened to the public. These GPS devices recorded the position of the instrumented vehicle at a frequency of 25 meters per location point registered between engine-on and engine-off events. These subjects remained instrumented for 8 weeks, during this time period the subjects followed their usual commute pattern without any instruction from the researchers. In addition, at the end of the study period (i.e. 8 weeks or 13 weeks depending on the GPS study), subjects completed a comprehensive final web-based survey to evaluate the driving experience on routes using different bridges choices, provide socio-demographic information (see Section 3.1), and also answer some questions regarding route preferences. 
A total of approximately 143 (about 46 by VMTINC, and 97 by University of Minnesota) subjects had usable (complete day-to-day GPS information) data required for this analysis. For this study, only 46 subjects ( 25 from VMTINC, and 21 from University of Minnesota) had the required data according to the subsequent Section 2.2.

\subsection{Methodology}

The GPS data analysis process can be divided in three phases:

1. Identification of commute trips per subject on the bridges of interest (see Figure 1);

2. Information extraction (e.g. travel time) of commute trips per subject;

3. Specification and estimation of a statistical model to determine the reasons for a subject to prefer the new I-35W bridge over other plausible alternatives.

The first phase utilizes the coordinates of the trips per subject, and the $T L G$ (defined in the subsequent paragraph) network in order to identify the trips crossing bridges, and the bridges crossed. This identification is done by spatial matching the coordinates of each bridge of interest to the coordinates of each set of trips for each subject. Also, subjects' trips must start at their home/work and end at their work/home locations in order to be considered commute trips (or more precisely direct commute trips as in trips without chaining behavior). The distance tolerance between origins (destinations) to home (work) locations was set to $600 \mathrm{~m}$. The home and work locations are geocoded (transformed into point coordinates) from the actual addresses provided by the subjects on the web-based surveys. The origin and destination pair of each trip is obtained by mapping the coordinate points into trajectories of engine-on and engine-off events. Moreover, inaccurate points due to GPS "noise", and out-of-town trips (e.g. during Thanksgiving) were excluded.

The $T L G$ network refers to a digital map maintained by the Metropolitan Council and The Lawrence Group (TLG). It covers the entire 7-county Twin Cities Metropolitan Area and is the most accurate GIS map of this network to date. The TLG network contains 290,231 links, and provides an accurate depiction of the entire Twin Cities network at the street level.

The second phase extracts usable information from the identified trips including: statistics of travel time distribution of all trips (both average and standard deviation) for each subject; total number of trips observed for each subject; and the frequency of routes (i.e. bridges) used by each subject. This process is performed for each time period of travel (e.g. AM), and for the period of interest (between September 18th and October 12th). On September 18th, the new I-35W Bridge opened to the public at $5 \mathrm{AM}$. On October 12th, the I-94 lanes were re-stripped, and consequently eliminating a traffic restoration measure implemented by MnDOT to ameliorate the bride collapse effects.

The third phase consists of fitting a statistical model to the data tabulated from the previous phases. The objective is to understand the factors behind the decision of commuters on whether to choose the new I-35W Bridge over other alternatives. This phase is described thoroughly in Section 4. 


\section{Descriptive Statistics}

2

\subsection{Socio-Demographics}

Table 1, summarizes socio-demographic information of the subjects. The sample differed somewhat from the population of the Twin Cities in several ways: subjects are older, more educated and have a more uniform distribution of income. Another characteristic of the sample is the variation of the subjects' time living at their current work and home location is high. In other words, the sample has subjects ranging from those living several years in their current work and/or home locations to those living a few months in their current work and/or home locations.

Table 1: Socio-Demographics attributes of the sample

\begin{tabular}{|l|l|l|l|}
\hline Number of Subjects & & 46 & \\
\hline Sex & Male & Sample & Twin Cities \\
& Female & $33.33 \%$ & $49.40 \%$ \\
\hline Age (Mean, Std. Deviation) & & $66.67 \%$ & $50.60 \%$ \\
\hline Education & 11th grade or less & $(50.35,10.49)$ & $(34.47,20.9)$ \\
& High School & $6.00 \%$ & $9.40 \%$ \\
& Associate & $33.06 \%$ & $49.60 \%$ \\
& Bachelors & $51.52 \%$ & $7.70 \%$ \\
& Graduate or Professional & $9.09 \%$ & $23.20 \%$ \\
& $\$ 49,999$ or less & $25.00 \%$ & $10.10 \%$ \\
\hline Household Income & $\$ 50,000$ to $\$ 74,999$ & $21.05 \%$ & $23.30 \%$ \\
& $\$ 75,000$ to $\$ 99,999$ & $30.26 \%$ & $14.60 \%$ \\
& $\$ 100,000$ to $\$ 149,999$ & $18.42 \%$ & $11.00 \%$ \\
& $\$ 150,000$ or more & $5.26 \%$ & $5.90 \%$ \\
\hline Race & Black/African American & $9.09 \%$ & $6.20 \%$ \\
& White or Caucasian & $69.70 \%$ & $87.70 \%$ \\
& Others & $21.21 \%$ & $6.10 \%$ \\
\hline Years at Current Work (Mean, Std. Deviation) & \multicolumn{2}{|l|}{} \\
\hline Years at Current Home (Mean, Std. Deviation) & \multicolumn{2}{|l|}{$(11.47,8.06)$} & \\
\hline
\end{tabular}

Twin Cities' Population statistics are obtained from the 2006-2008 American Community Survey

3-Year Estimates, Minneapolis-St. Paul-Bloomington, MN-WI Metropolitan Statistical Area, Retrieved November 25, 2009. (n.d.)

\subsection{Routes: Preferences, and Attributes according to survey data}

Figure 2 presents the bridges rankings according to the subjects responses in the final web-based survey. The I-35W Bridge is the most preferred. This is not coincidental, as many subjects were selected based on whether I-35W would be a component of a shortest route to work. It should be noted that this preference is marked after the I-35W bridge reopened. Furthermore, the high preference for I-35W bridge agrees with the subjects stated reasons for choosing a route (Figure 3 ). The two most important reasons for choosing a route indicated by the subjects are travel time, travel 


\section{3.3 Route Changing Behavior according to survey data}

5 In Tables 2 and 3, the subjects stated that they were prone to try alternative routes, and/or to

time predictability, travel distance and other reasons unique to the subjects. The travel distance is an interesting reason as subjects are likely to drive to the bridges closer to their home and work location. Bridges that are farther might not attract subjects. change routes (if justified) after the I-35W Bridge reopened. The most cited (41\%) reason the subject's indicated for changing routes is that plausible alternatives have shorter travel times. In contrast, $45 \%$ of subjects who did not change routes considered that the alternatives were not better. This change of routes probably was required as many subjects did not reduce the number of river crossings according to Table 4, and thus alternatives to I-35W had to be found. In addition, it should be noted that subjects are asked whether they tried alternative routes irrespective to them changing routes, and vice versa.

Table 2: Route changed after I-35W Bridge Reopen

\begin{tabular}{|lll|}
\hline Number of Subjects & 46 & \\
\hline Usual route changed after I35W Bridge Reopening & Yes & $62.60 \%$ \\
& No & $37.50 \%$ \\
\hline Reasons for changing route & Old route is more congested now. & $9.09 \%$ \\
& New route has a shorter travel distance. & $9.09 \%$ \\
& New route has a shorter travel time. & $40.91 \%$ \\
& The travel time of new route is more reliable & $31.82 \%$ \\
& (predictable) & \\
& Other & $9.09 \%$ \\
\hline
\end{tabular}

Table 3: Alternative routes after I-35W Bridge Reopen

\begin{tabular}{|lll|}
\hline Number of Subjects & 46 & \\
\hline Tried Alternative Routes other than usual routes & Yes & $33.64 \%$ \\
after I35W Bridge Reopened & No & $36.36 \%$ \\
\hline Reasons for not changing route & No alternative for my route to work. & $20.00 \%$ \\
& Apathetic about looking for alternatives. & $0.00 \%$ \\
& The alternative routes are not likely to be better & $45.00 \%$ \\
& off. & \\
& The time and effort of trying alternatives & $25.00 \%$ \\
& outweighs possible time savings. & \\
& Other & $10.00 \%$ \\
\hline
\end{tabular}


Table 4: Crossing-river trips after I-35W Bridge Collapse

\begin{tabular}{|lll|}
\hline Number of Subjects & 46 & \\
\hline Fewer crossing-river trips were made after I35W Bridge Collapse & Yes & $12.12 \%$ \\
& No & $87.88 \%$ \\
\hline Frequency of crossing-river trips cancelled/consolidated with other trips. & Several trips per day. & $0.00 \%$ \\
& Several trips a week. & $0.00 \%$ \\
& Once a week. & $37.50 \%$ \\
& Once a month. & $25.00 \%$ \\
& Less than once a month. & $37.50 \%$ \\
\hline
\end{tabular}

\section{Statistical Model}

2 A statistical model using weighted least-squares (WLS) logit is used to predict the proportion of 3 I-35W trips performed by a traveler. A WLS logit analyzes binary or dichotomous choices, and these choices can be weighted by a frequency (number of I-35W trips per subject in this case). The reader can refer to Trivedi and Cameron (2005), and Ruud (2000) for additional details about weighted least square estimators, and logit models.

The proposed model studies the bridge swapping behavior of commuters (i.e. choosing I-35W Bridge vs. Other alternatives). The dependent variable is represented by the proportion of trips traveled on the new I-35W Bridge out of a subject's total trips during the period of interest (September 18th and October 12th). The other portion of trips consist of other bridge alternatives frequented by the commuters in the study such as: I-94, I-694, Lowry Avenue, Cedar Ave (19th Avenue - 10th Street), Hennepin Ave, Washington Ave, Franklin Ave, and others.

The specification of the WLS logit is as follows:

$$
\mathbf{L} \sim \mathbf{f}\left(\mathbf{T}_{\mathbf{m}}, \overline{\mathbf{T}}_{\text {I-35W}}, \mathbf{V}_{\text {I-35W }}, \overline{\mathbf{T}}_{\text {Alternatives }}, \mathrm{V}_{\text {Alternatives }}, \mathbf{D}_{\text {Alternatives }}, \mathbf{S}\right)
$$

where:

- L : Proportion of I-35W trips

- $\mathbf{T}_{\mathbf{m}}$ : Time Period - The time of day. It is 1 for PM, and 0 for AM.

- $\overline{\mathbf{T}}_{\mathbf{I}-35 \mathrm{~W}}$ : I-35W: Average Travel Time - The average (arithmetic mean) travel time experienced by each subject while driving on the new I-35W between September 18th and October 12th. (minutes).

- $\mathrm{V}_{\text {I-35W }}$ : I-35W: Travel Time Variability - The standard deviation of the travel time experienced by each subject while driving on the new I-35W between September 18th and October 12th. In addition, it also limits the number of subjects in the sample, because the subjects must have at least two trips performed on the I-35W bridge. (minutes).

- $\overline{\mathbf{T}}_{\text {Alternatives }}$ : Alternatives: Average Travel Time - The average (arithmetic mean) of the travel time experienced by each subject on all other bridge alternatives excluding the new I-35W bridge. This average also includes trips before September 18th (but not after October 12th) as certain subjects did not travel on any other alternatives after the new bridge reopened. In this way, a measure of the possible travel time for those subjects can be calculated without having to reduce further the sample size. (minutes). 
- $\mathrm{V}_{\text {Alternatives }}$ : Alternatives: Travel Time Variability - The standard deviation of the travel time experienced by each subject, while driving all other bridge alternatives excluding the new I-35W bridge. This standard deviation also includes trips before September 18th (but not over October 12th) as certain subjects did not travel on any other alternatives after the new bridge reopened. (minutes).

- $\mathrm{D}_{\text {Alternatives }}$ : Alternatives: Bridge Diversity - The number of distinct alternatives (bridges) a subject used from September 18th (and before) to October 12th.

- S : Socio-Demographic variables - These are extracted from the socio-demographic questions in the web-based surveys.

- Gender (1 = Male; 0 = Female).

- Income. Four categories: $(\$ 0, \$ 49,999],(\$ 50,000, \$ 74,999],(\$ 75,000, \$ 99,999]$, and $(\$ 100,000, \infty+)]$. The first category is the base case. (2008 US dollars).

The explanatory variables are based on section 3.2. The subjects indicated travel time, and travel time predictability as important variables for their bridge preference. In addition, the diversity variable is included as a proxy in order to account for a subject's alternative search behavior; some subjects actively searched for alternatives, while others did not. The socio-demographic variables are included to handle observed heterogeneity in the sample; bridge choice preferences unique to groups segmented by either gender and/or income categories.

\section{Results}

Table 5 shows the parameter estimates for the specified model. Factors found statistically significant include: average travel time, travel time variability, bridge diversity, and socio-demographic variables. This corroborates Figure 3 as it indicates travel time as an important factor for the subjects. In terms of goodness of fit, the model has a $R^{2}$ of 0.5865 . Furthermore, the results presented by the regressors are:

\subsection{Time Period}

This variable was not found statistically significant, and thus the proportion of the I-35W bridges for AM and PM did not seem to be systematically different.

\subsection{I-35W: Average Travel Time and Travel Time Variability}

The average travel time and travel time variability of the I-35W bridge were found statistically significant. Both have the expected sign; high travel time and high travel time variability of I-35W should lead to smaller proportion of trips using I-35W. In addition, it agrees with Table 2 as smaller average travel time and higher travel time predictability (low variability) for I-35W should attract possible commuters looking for new alternatives. 


\subsection{Alternatives: Average Travel Time and Travel Time Variability}

The average travel time and travel time variability of the alternative bridges (excluding I-35W) were found statistically significant. Both have the expected sign; high travel time and high travel time variability of alternatives to I-35W should lead to higher proportion of trips using I-35W. However, the travel time variability was less significant than its I-35W counterpart. This is perhaps product of the aggregations of different bridge alternatives.

\subsection{Alternatives: Bridge Diversity}

This variable was found statistically significant. It indicates that the more distinct alternatives a subject experience, the lower will be the subject's proportion of trips on the I-35W bridge. A possible reason for this result is that travelers may still be in the process of searching for their best alternative (I-35W or other) according to their own criteria.

\subsection{Socio-Demographic variables}

Neither of the specified socio-demographic variables were found statistically significant. The choice situation tended to be dominated by the measures of the travel time distributions.

Finally, other factors not included as pointed by the subjects in Table 3 may influence their preferred bridge choice, even if travel time benefits are present.

Table 5: Weighted Least-Squares Logit for I-35W Choice

\begin{tabular}{|c|c|c|c|c|c|}
\hline Number of Subjects & 46 & Estimate & Std. Error & T-Statistic & P-Value \\
\hline Time Period & $\mathbf{T}_{\mathrm{m}}$ & -0.229 & 0.234 & -0.98 & 0.331 \\
\hline I-35W: Average Travel Time ${ }^{a}$ & $\overline{\mathbf{T}}_{\mathbf{I}-35 \mathrm{~W}}$ & -0.0807 & 0.0171 & -4.73 & $0.000 * * *$ \\
\hline I-35W: Travel Time Variability ${ }^{b}$ & $V_{I-35 W}$ & -0.0905 & 0.0287 & -3.16 & $0.002 * * *$ \\
\hline Alternatives: Average Travel Time ${ }^{a}$ & $\overline{\mathbf{T}}_{\text {Alternatives }}$ & 0.0732 & 0.0126 & 5.83 & $0.000 * * *$ \\
\hline Alternatives: Travel Time Variability ${ }^{b}$ & $\mathrm{~V}_{\text {Alternatives }}$ & 0.0505 & 0.0298 & 1.70 & $0.095^{*}$ \\
\hline Alternatives: Bridge Diversity & $\mathrm{D}_{\text {Alternatives }}$ & -0.309 & 0.182 & -1.70 & $0.094 *$ \\
\hline Gender $(1=$ Male; $0=$ Female $)$ & $\mathrm{G}_{\mathrm{M} / \mathrm{F}}$ & -0.240 & 0.193 & -1.24 & 0.218 \\
\hline Income $[\$ 50,000, \$ 74,999](1=\mathrm{In} ; 0=$ Out $)$ & $\mathrm{I}_{50 / 74}$ & 0.182 & 0.264 & 0.69 & 0.495 \\
\hline Income $[\$ 75,000, \$ 99,999](1=\mathrm{In} ; 0=$ Out $)$ & $I_{75 / 99}$ & 0.359 & 0.224 & 1.60 & 0.113 \\
\hline Income $[\$ 100,000, \infty+)(1=\mathrm{In} ; 0=$ Out $)$ & $I_{100}$ & 0.387 & 0.245 & 1.09 & 0.281 \\
\hline (Intercept) & & 0.350 & 0.322 & 1.09 & 0.281 \\
\hline R-Squared & $R^{2}$ & 0.5865 & & & \\
\hline Adj. R-Squared & $\operatorname{Adj}-R^{2}$ & 0.5248 & & & \\
\hline Root Mean Square Error & RMSE & 0.7045 & & & \\
\hline
\end{tabular}

$*$ is $10 \%$ significance level, $* *$ is $5 \%$ significance level, $* * *$ is $1 \%$ significance level

${ }^{a}$ It is the arithmetic mean of the travel time distribution of the trips for the mentioned period of study.

${ }^{b}$ It is the standard deviation of the travel time distribution of the trips for the mentioned period of study. 


\section{Discussion and Limitations}

2 In summary, the main results (see section 5) of the model indicate that the average travel time, and 3 the travel time variability are the key factors for bridge choice preference across subjects. These results should be understood from an aggregate level. The measures of centrality (average) and dispersion (standard deviation) of the travel time distributions for the I-35W bridge (i.e. all trips within the time period as defined in section 4) per subject, and the alternatives bridges (i.e. all trips within the time period as defined in section 4) per subject are significantly different per subject (that is to say that each subject is a record in the dataset). This difference could be that some subjects for the whole travel time distribution (across the trips for the whole period) may have experienced on average higher travel time for I-35W in comparison to their plausible alternatives, or vice versa. Moreover, it means that it is assumed subjects at the aggregate level "settled" for a particular bridge choice. However, this has the side effect of neglecting that subjects are actually updating their decision most likely at a day to day level. In other words, subjects may have found better (worse) alternatives as soon as possible (early or late during the time period), and proceeded to change (stay) at their current choice. Therefore, the number of trips for either I-35W or the plausible alternatives may exhibit an state dependency effect (previous choices influence future choices; experience factor). Furthermore, this adaptation process (selecting choices from previous experience) is likely to happen regardless of whether a network disruption occurred, but a disruption (depending on its temporal duration and its spatial occupancy) in principle will generate the traffic conditions (e.g. aggravate the differences across the choices' travel times) that will motivate travelers to change and/or try new alternatives.

Another important aspect is the searching behavior of the subjects (implied in the previous paragraph). The alternatives diversity variable was included to distinguish between subjects that tried alternative bridges vs. subjects that did not try any alternative bridges. Therefore, the variable acts as a "proxy" for search behavior. However, it is obvious that subjects with bridge diversity higher than zero will have less trips for the I-35W choice. This is because only direct commute trips are considered, and thus on regular working schedules (as those required for this study) the number of commute trips is likely to not change ( 2 commute trips per day) significantly from day to day. Therefore, the diversity variable has the correct sign and effect (higher values should reduce the number of I-35W trips), but it does not capture the feedback behavior (i.e. willingness and inertia to search for alternatives; see Table 3) of the subjects.

Furthermore, the model benefits from the GPS data due to its detailed commute level information, despite that fact that the final sample's characteristics differs from the Twin Cities' characteristics. Thus, this limits the level of applicability of the model at the metropolitan level. In addition, other socio-demographic variables (e.g. household size) may indicate heterogeneity in the sample, despite the fact that such heterogeneity was not found at statistically significant levels.

Finally, readers should be reminded of the exploratory nature of the study, and in this regard the model does identify the important factors of the bridge choice process, despite not taking into account state dependency (experience factor), search behavior, and other factors explicitly. 


\section{Conclusion}

Network disruptions force travelers to adapt by changing to other modes, finding alternative routes, canceling/consolidating trips, rescheduling trips, and in severe cases look for new residential and/or work locations. However, questions arise about the effects after the disruption, and also about the influences of traffic restorations done by DOTs to the traffic patterns in the network. In the case of the I-35W Bridge collapse, MnDOT performed two major changes to the network: the opening of a new I-35W bridge, and the re-stripping of I-94 in order to have additional lanes. In this study, an exploratory analysis was performed focusing solely on the factors behind the travelers selection of the new I-35W bridge over their previously available alternatives after its collapse. A proposed model following (WLS logit) was formulated to identify the magnitude and direction of the contributions of elements such as travel time in the bridge choice process during this transition period.

According to the survey data (Tables 2 and 3), subjects with at least two trips on the new I-35W bridge (the selected sample size) stated a high willingness to try new alternatives, and indicated that their usual route changed. Furthermore, travel time and travel time predictability (low variability) were selected as the main reasons for trading routes. This result also agreed with the bridge choice model fitted to the GPS data of the same subjects surveyed. Therefore, travel time savings and reliability were the key components regardless of their socio-demographic differences in explaining their swapping behavior (I-35W vs. Other alternatives). However, resistance (e.g. route constraints, high search costs) to choose the new I-35W bridge or other alternatives was also present as stated by the subjects.

Future research is required as very few studies have extensively covered major disruptions, because naturally they are hard to predict, and thus data is not collected. In this case, the GPS data acquired is an invaluable scientific resource that allows further exploration with distinct model formulations. A possible path for new research is the development of models accounting for the experience factor (state dependency). This could be analyzed by considering the duration of memory of travel times - how far back in time (1 week, 2 weeks, 3 weeks) travelers remember average travel times for a specific route they followed. This experiential model could be helpful, because it might identify the beginning of the bridge (or route) changing process.

\section{Acknowledgements}

This study is supported by the Oregon Transportation Research and Education Consortium (2008130 Value of Reliability and 2009-248 Value of Reliability Phase II) and the Minnesota DOT project "Traffic Flow and Road User Impacts of the Collapse of the I-35W Bridge over the Mississippi River". We would also like to thank Kathleen Harder, John Bloomfield, and Shanjiang Zhu. 


\section{References}

2006-2008 American Community Survey 3-Year Estimates, Minneapolis-St. Paul-Bloomington, MN-WI Metropolitan Statistical Area, Retrieved November 25, 2009. (n.d.).

URL: http://factfinder.census.gov/

Chang, S. and Nojima, N. (2001), "Measuring post-disaster transportation system performance: the 1995 kobe earthquake in comparative perspective", Transportation Research Part A, Vol. 35, pp. 475-494.

Dimitriou, D., Karlaftis, M., Kepaptsoglou, K. and Stathopoulos, M. (2006), Public transportation during the athens 2004 olympics: From planning to performance., in 'Proceedings of the 85th Transportation Research Board Annual Meeting, Washington D.C., U.S.A.' .

Ferguson, E. (1992), “Transit ridership, incident effects and public policy", Transportation Research Part A, Vol. 26, pp. 393-407.

Giuliano, G. and Golob, J. (1998), "Impacts of the northridge earthquake on transit and highway use", Journal of Transportation and Statistics , Vol. 1, pp. 1-20.

Hensher, D. and Brewer, A. (2002), "Going for gold at the sydney olympics: how did transport perform?”, Transport Reviews, Vol. 22, pp. 381-399.

Hunt, J., B. A. and Stefan, K. (2002), "Responses to centre street bridge closure: Where the disappearing travelers went”, Transportation Research Record, Vol. 1807, pp. 51-58.

Lo, S. and Hall, R. (2006), "Effects of the los angeles transit strike on highway congestion", Transportation Research Part A, Vol. 40, pp. 903-917.

Ruud, P. (2000), An introduction to classical econometric theory, Oxford University Press.

Trivedi, P. K. and Cameron, A. C. (2005), Microeconometrics: Methods and Applications, Cambridge Univ. Press.

Tsuchida, P. and Wilshusen, L. (1991), "Effects of the 1989 loma prieta earthquake on commute behavior in santa cruz county, california", Transportation Research Board. , Vol. 1321, pp. 2633.

van Exel, N. and Rietveld, P. (2001), "Public transport strikes and traveller behaviour", Transport Policy, Vol. 8, pp. 237-246.

Wesemann, L., Hamilton, T., Tabaie, S. and Bare, G. (1996), "Cost-of-delay studies for freeway closures caused by northridge earthquake", Transportation Research Record, Vol. 1559, pp. 6775.

Zhu, S., Levinson, D., Liu, H. and Harder, K. (2010), "The traffic and behavioral effects of the i-35w mississippi river bridge collapse", Transportation Research part A, Vol. 44, pp. 771-784. 


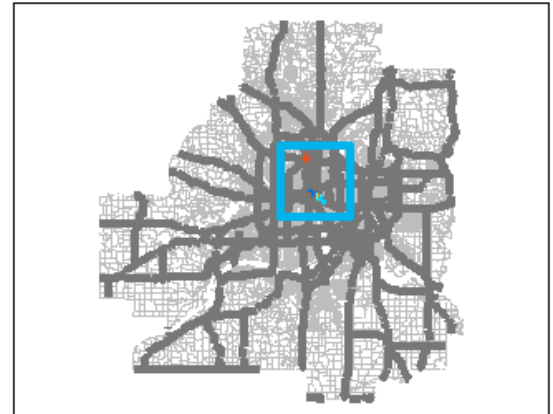

\section{Legend}

I-694

- Franklin Ave

I-94

Hennepin Avenue

3rd Avenue

Washington Avenue - Cedar Avenue

I-35W

MSP Freeway System

\section{neXus}

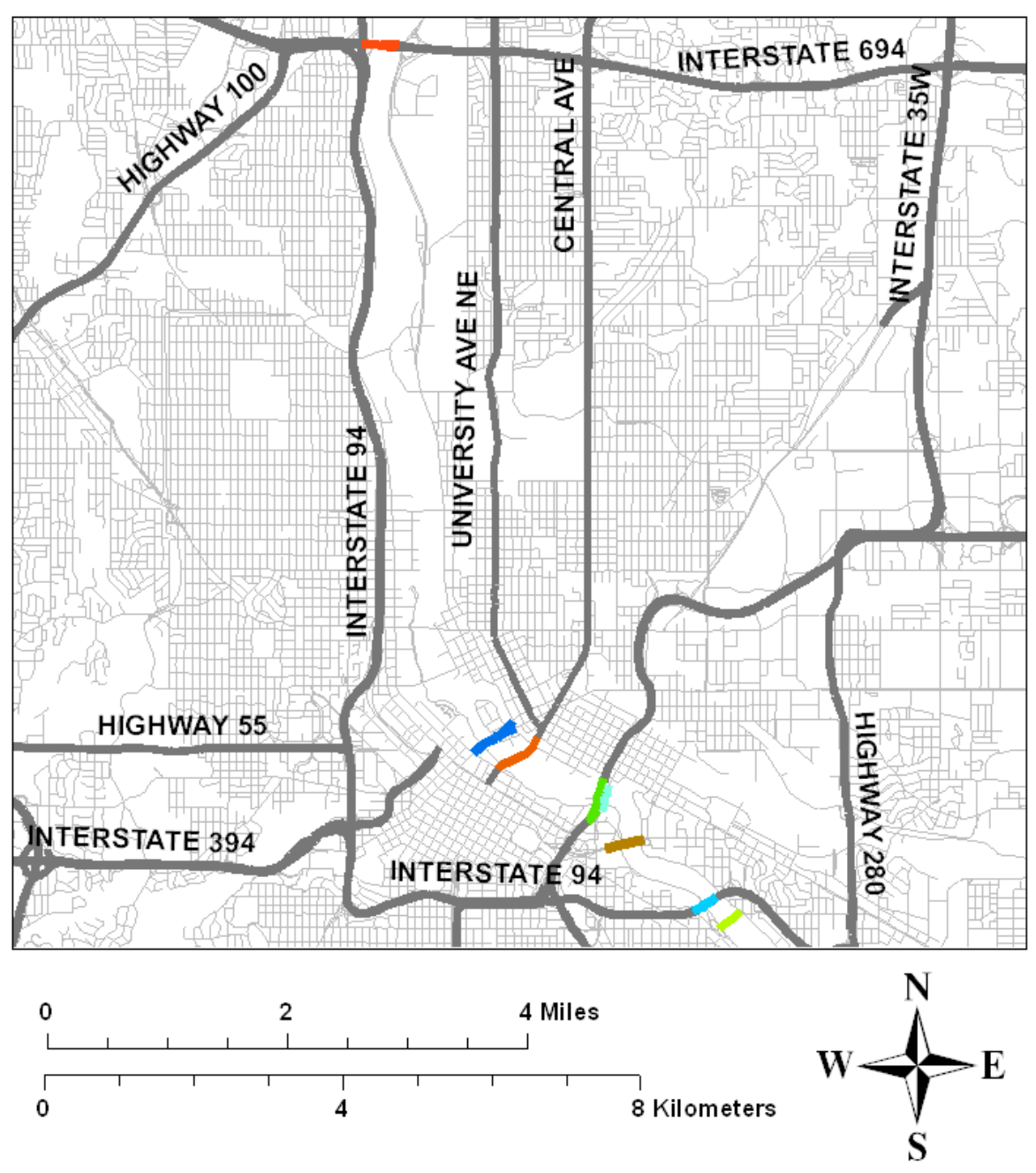

Figure 1: Bridge Locations 
Figure 2: Routes Preference Top 3 Rank

Route Preferences Ranking (H2W)

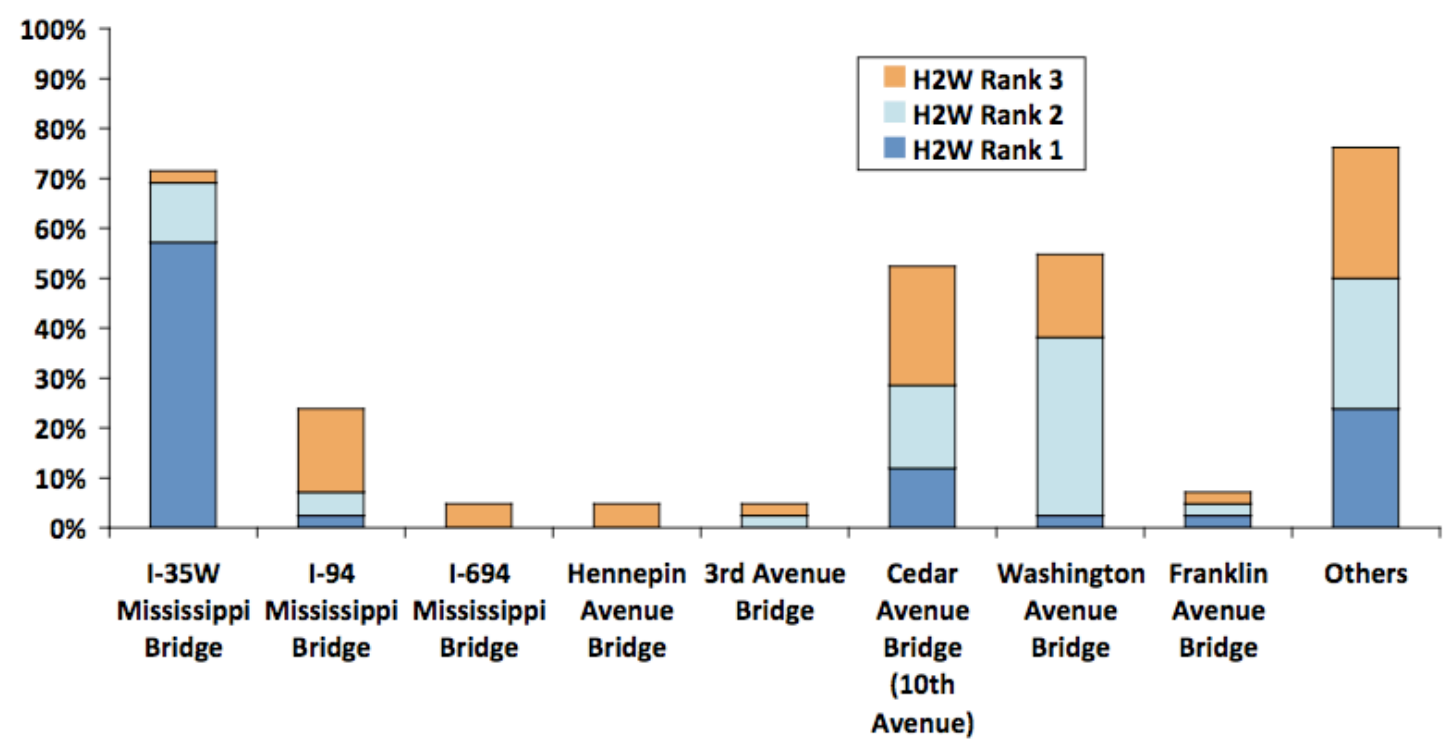

Route Preferences Ranking (W2H)

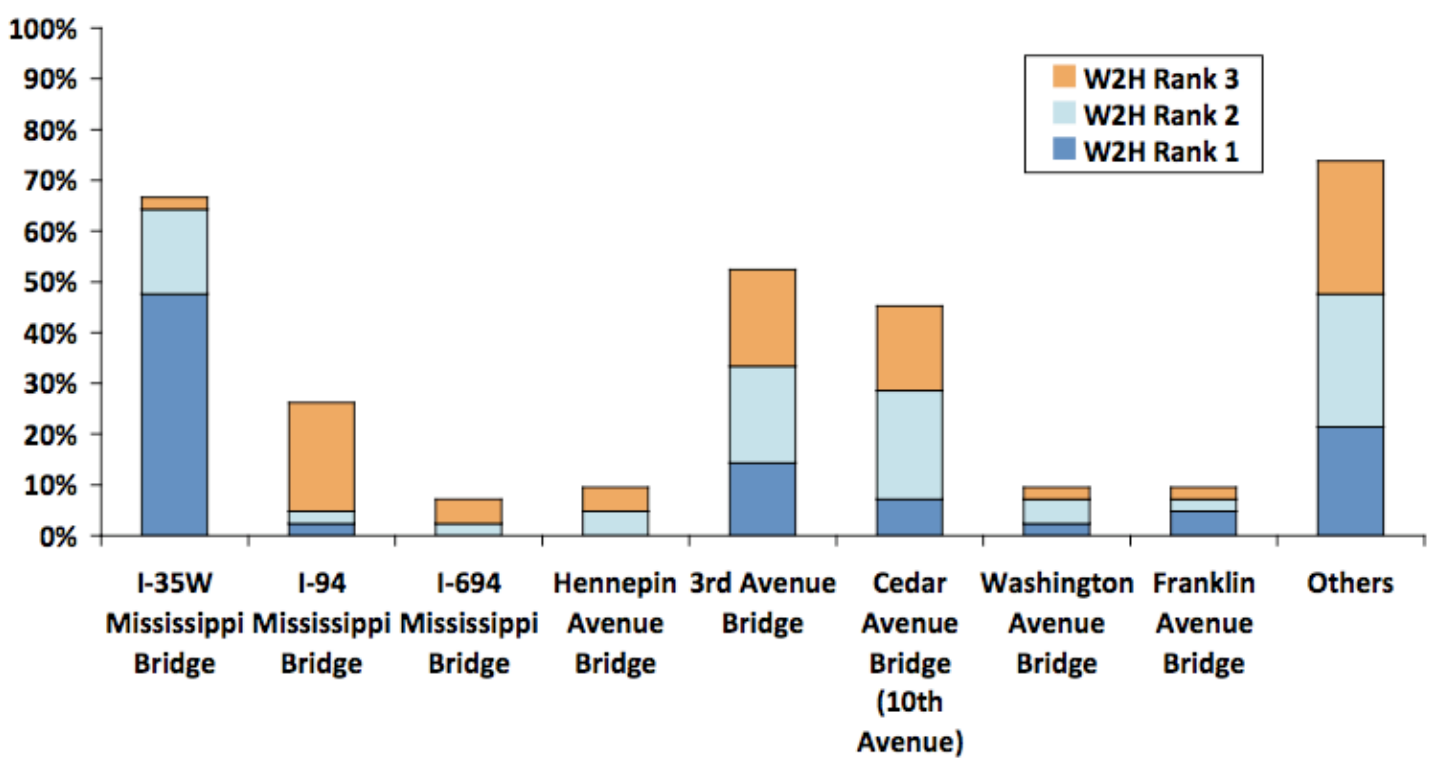


Figure 3: Reason behind route preferences Top 3 Rank

Reasons behind route preferences ranking (H2W)

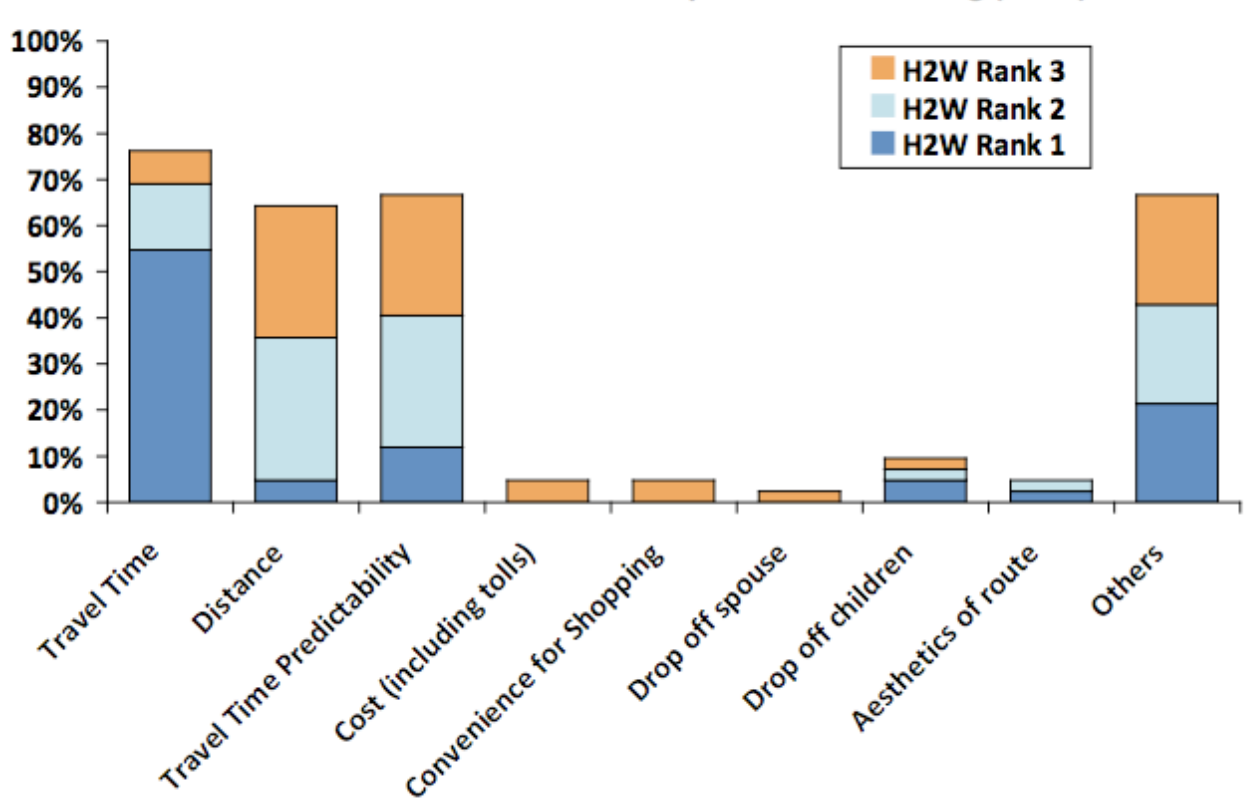

Reasons behind route preferences ranking (W2H)

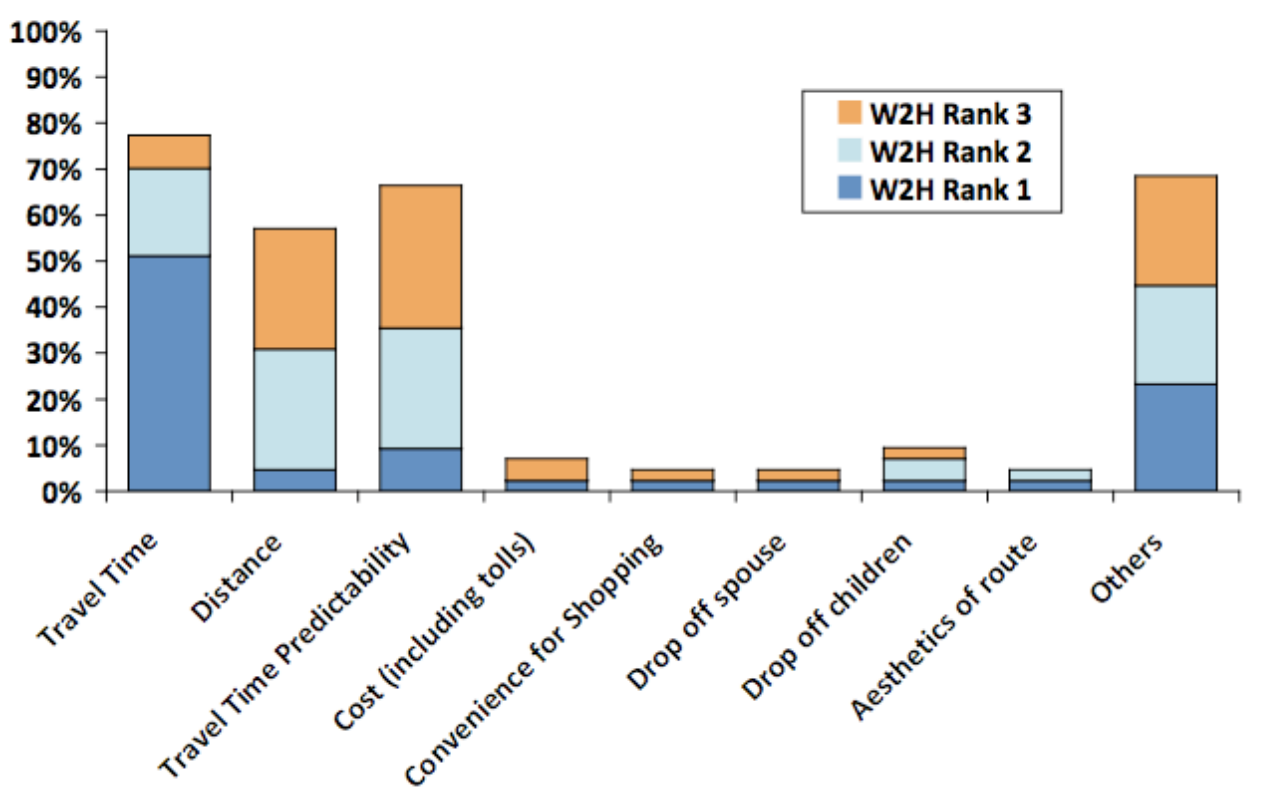

